Universidad Nacional de La Plata

Facultad de Humanidades y Ciencias de la Educación

Departamento de Sociología

\title{
Comprender la ciudad desde sus habitantes. Relevancia de la teoría de prácticas sociales para abordar la movilidad
}

\author{
Understanding the city from its dwellers' perspective. The \\ relevance of social practice theory to approach mobility
}

\section{Paola Jirón* y Carlos Lange*}

* Instituto de la Vivienda (INVI) - Facultad de Arquitectura y Urbanismo Universidad de Chile, Chile | paolajiron@uchilefau.cl, clange@uchilefau.cl

\section{PALABRAS CLAVE}

Prácticas de Movilidad

Aprendizaje urbano

Saberes y conocimiento urbano

KEYWORDS

Mobility practices

Urban learning

Urban knowledge

\section{RESUMEN}

Transantiago, el sistema de transporte público de la ciudad de Santiago, ha generado diversas críticas en cuanto al poco reconocimiento por parte de los planificadores urbanos y de transporte de la relevancia que tienen las prácticas de movilidad en la vida cotidiana y por ende en los sistemas de transporte urbano. Desde su implementación en 2007 hasta la actualidad, sus usuarios han podido reaprender a viajar, crear nuevas espacialidades por medio de nuevas prácticas sociales y subvertir algunos procesos por medio de la transformación de ciertas prácticas. Lo anterior permite establecer la existencia de unestrecho vínculo entre las prácticas sociales de movilidad, sus procesos cotidianos de aprendizaje y la producción de conocimientos y saberes relevantes y necesarios para comprender la ciudad integrando las perspectivas de habitantes e interventores. El presente artículo plantea que el estudio de las prácticas sociales urbanas constituye un camino relevante para comprender y promover el rol de los habitantes urbanos en el desarrollo de sus territorios.

\section{ABSTRACT}

Transantiago, the city of Santiago's public transport system, has generated multiple criticisms in terms of the limited recognition from urban and transportplannerson the relevance mobility practices have on daily life and thus on urban transport systems. Since its implementation in 2007, travellers have had to relearn to travel, create new spatialitythrough new practices and subvert processes thoughthe transformation of specific practices. This helps to establish the existence of a close link between mobility socialpractices, daily learning processes from these practices and the production of relevant and necessary knowledge to understand the city by integrating dwellers' and planners' perspectives. This paper proposesthe study of urban social practices as one way of understanding the role urban dwellers have on spatial development. 


\section{Introducción}

Una crítica constante a los procesos de desarrollo urbano es que aquellos que planifican e intervienen la ciudad poco o nada se vinculan con aquellos que la habitan, transforman y crean día a día (de Certeau, 1986; Lefebvre, 1972 y Healy, 2003). Si bien en la actualidad existen distintas estrategias para acercar a los habitantes urbanos al quehacer planificador, la existencia de intervenciones urbanas en las cuales no se reconoce el aporte que éstos pueden realizar en dichos ámbitos es aún generalizada. Asimismo, tampoco se observan procesos de aprendizaje sistemático sobre las intervenciones urbanas, aunque muchas de ellas exijan revisión y autocritica en su implementación.

Un ejemplo de lo anterior es la implementación y funcionamiento de Transantiago, el sistema de transporte público de la ciudad de Santiago. Éste constituye un caso interesante de estudio por cuanto la falta de reconocimiento a la relevancia que las prácticas de movilidad tienen en la vida cotidiana (Jirón, 2012 y Ureta, 2015) ha sido responsable en gran medida de que su implementación sea considerada un gran fracaso de la política de transporte y urbana en Chile (Alamo, 2008).

Frente a este diagnóstico el presente artículo plantea que el estudio de las prácticas sociales urbanas constituye un camino relevante para comprender y promover el rol de los habitantes urbanos en el desarrollo de sus territorios. En el caso de Transantiago, desde su implementación en 2007 hasta la actualidad, sus usuarios han podido reaprender a viajar, crear nuevas espacialidades por medio de nuevas prácticas sociales y subvertir algunos procesos por medio de la transformación de ciertas prácticas. Lo anterior permite establecer la existencia de unestrecho vínculo entre las prácticas sociales de movilidad, sus procesos cotidianos de aprendizaje y la producción de conocimientos y saberes relevantes y necesarios para comprender la ciudad integrando las perspectivas de habitantes e interventores.

\section{Relevancia del enfoque de prácticas sociales}

En términos generales, las prácticas sociales pueden entenderse como los distintos modos de hacer que las personas desarrollamos en nuestras vidas cotidianas, las cuales nos permiten establecer relaciones sociales y configurar el espacio habitado. El carácter cotidiano de las prácticas sociales alude a aquellas condiciones diarias de vida, es decir, los modos como vivimos, trabajamos, consumimos, nos relajamos, nos relacionamos con otros, forjamos nuestras identidades, etc. En tal sentido, el concepto de cotidianeidad enfatiza las interacciones entre prácticas individuales y estructuras sociales, entre distintos tipos de acciones y diferentes niveles de conciencia.

Las prácticas cotidianas en el espacio urbano pueden servir para mediar entre habitantes y grupos sociales por un lado y estructuras más amplias e instituciones de la sociedad por otro (Vaiou \&Lykogianni, 2006). Como ya lo plantearon autores como Lefebvre (1972) y de Certeau (1986), reconocer la importancia de la cotidianeidad en las prácticas sociales de los habitantes urbanos permite explorar aquellos aspectos invisibles y muchas veces problemáticos del habitar urbano, que muchas veces son ignorados por los tomadores de decisiones sobre intervenciones urbanas en el territorio. 
Una perspectiva fundamental para comprender la estrecha relación entre prácticas sociales y cotidianeidad se daa través del concepto de habitus formulado por Bourdieu (1998). Para éste, la cotidianeidad permite reconocer en las prácticas de habitar tanto un carácter implícito como explícito. En el caso de las primeras, éstas actúan como un tipo de saber encorporado, es decir no reflexivo, el cual se reproduce de forma repetitiva y automática produciendo y reproduciendo el espacio habitado y constituyendo un campo de experiencias cotidianas. Una vez identificadas y sistematizadas, éstas se vuelven reflexivas, y por tanto explícitas en cuanto a su relevancia.

Muchos de los aspectos mencionados anteriormente adquieren continuidad en las actuales vertientes de la teoría de prácticas sociales, las cuales enfatizan la importancia de comprender cómo prácticas específicas espacializadas influyen en la vida cotidiana. Pese a que existen discrepancias respecto a si existe o no una "teoría de prácticas”, autores como Schatzki (1996, 2001 y 2012), Reckwitz (2002) y Warde (2004 y 2005) han identificado distintos componentes que permiten delinear un enfoque común articulado en torno al interés en "lo cotidiano" y en "el mundo de la vida”, y reconocen el origen de este enfoque teórico en autores como Bourdieu y Giddens, entre otros. En este marco, las prácticas son concebidas como un despliegue de actividades humanas encorporadas y mediadas materialmente, organizadas en torno a entendimientos compartidos.

Desde la perspectiva de Schatzki (2001 y 2002), las prácticas pueden entenderse como un conjunto de modos de hacer y decir concretos que ponen en acción los agentes para resolver determinados requerimientos. Para Reckwitz (2002), una práctica puede ser un comportamiento de tipo rutinario que se compone de elementos interconectados: formas de actividades corporales, formas de actividades mentales, 'cosas' y su uso, un conocimiento histórico en la forma de una comprensión, un saber hacer, estados de emoción y conocimiento motivacional. En síntesis, las prácticas sociales permiten comprender el comportamiento habitual y cotidiano de las personas..

La particular preocupación e interés que los enfoques de prácticas tienen por la experiencia cotidiana de las personas permite abrir nuevas vías de comprensión sobre el espacio urbano habitado. Ello es así porque el reconocimiento de las particularidades de los distintos territorios urbanos y de las condiciones de vida de sus habitantes obliga a cuestionar la conveniencia de los modelos de desarrollo urbano actualmente vigentes, muchos de los cuales resultan inapropiados y descontextualizados.

En complementariedad con esta perspectiva, resulta importante destacar también los aportes generados desde la sociología pragmática para comprender la relevancia de las prácticas sociales. También surgida en torno a las propuestas desarrolladas por Bourdieu en torno al concepto de habitus, se constituyen en revulsivas de éste promoviendo nuevas formas de entender la importancia de la reflexividad asociada a las prácticas sociales.

Esta perspectiva permite atender la relevancia de las prácticas en situaciones concretas de desarrollo, donde los habitantes urbanos desarrollan disposiciones particulares y distintivas frente al funcionamiento institucional. En tal sentido, esta perspectiva permite avanzar en la comprensión de la "acción situada”, en torno a la cual los actores desarrollan competencias en función del curso de acción de las situaciones en las cuales se desenvuelven cotidianamente (Corcuff, 2014). Estas competencias les permiten a los actores desenvolverse diferenciadamente según los "regímenes de 
acción” en los cuales éstos participan.

Particularmente interesante resulta la perspectiva de L. Thévenot (2016), quien enfatiza en la relevancia de investigar pormenorizadamente las prácticas sociales. Por una parte, su consideración permite reducir la distancia entre el conocimiento experto desarrollado por especialistas y aquél conocimiento desarrollado por los habitantes sustentado en sus prácticas sociales cotidianas. Asimismo, la atención sobre las situaciones en acción permite develar cómo ambas esferas de comprensión de la realidad se organizan a partir de una interacción y entrelazamiento constante. Por último, ello permite acceder a un análisis situado de las interrelaciones entre los distintos actores y agentes de la movilidad urbana cotidiana, y de las distintas “competencias” que éstos aprenden y usan en situaciones concretas, propiciando una constante creación de nuevas formas de acción posibles dentro del marco de una situación.

En tal sentido, y siguiendo a Nardacchione (2016), es posible afirmar que atender a las prácticas sociales de los habitantes permite comprender cómo éstos aprenden y adquierencompetencias prácticas sobre el funcionamiento de los sistemas urbanos, considerando incluso que muchas de sus prácticas y dispositivos ponen en cuestión la institucionalidad asociada a la movilidad urbana cotidiana, transformándola.

Lo anterior posee también una relevancia de carácter metodológico. Tal como plantean Barthe et al. (2016), comprender la acción situada requiere de un acercamiento y de un acompañamiento a los habitantes urbanos que posibilite una descripción constante, pormenorizada y sutil de sus prácticas, por cuanto éstas no pueden ser consideradas nunca totalmente completas sino más bien en permanente producción. Este requerimiento constituye unimportante antecedente para considerar la conveniencia de un abordaje etnográfico en torno a su producción.

\section{Prácticas cotidianas de movilidad: la vida después de Transantiago}

Una de las áreas donde la teoría de prácticas ha sido particularmente beneficiosa para la comprensión del espacio urbano habitado en la ciudad de Santiago ha sido el de la movilidad urbana cotidiana. En los últimos años, la importancia de la movilidad cotidiana ha sido reconocida entre académicos y profesionales como particularmente influyente en la efectiva transformación del espacio urbano. Tal como lo menciona Cresswell (2010) la movilidad implica comprender el movimiento, así como la experiencia y significado de los viajeros.

No obstante ello, la importancia de las experiencias de movilidad aún son escasamente visibilizadas y reconocidas por quienes definen e implementan políticas urbanas y de transporte, particularmente en ciudades de países en desarrollo. Lo anterior se ve ejemplificadoen el caso de Transantiago, el sistema de transporte público de la ciudad de Santiago implementado en el año 2007. Este implicó la reestructuración completa de la red pública de transporte existente a partir de dos conceptos principales: complementación e integración: mientras el primero se orientaba a complementar el uso del autobús con el sistema de metro existente (y extender la red de metro), el segundo incorporaba una tarifa única tanto para el metro como los autobuses. Además, se introdujo una nueva tarjeta de viaje inteligente (Tarjeta Bip!) con el fin de facilitar el pago y hacer el viaje más seguro (MOPTT, 2004). De esta forma, se esperaba que una integración mejorada y una mayor 
coordinación entre operadores y modos de transporte público reducirían los gastos de los hogares que vivían en la periferia, mejorando simultáneamente la accesibilidad.

Sin embargo, Transantiago comenzó su operación sin contar con todos los sistemas técnicos, financieros, de información e infraestructura adecuados de funcionamiento, lo cual generó tres problemas principales desde un punto de vista de la movilidad: la red, la infraestructura y la comunicación $\underline{\underline{1}}$, modificando completamente el sistema de transporte público capitalino. Esto tuvo serios impactos en la vida cotidiana de los viajeros, muchos de los cuales se adaptaron al sistema mientras que otros lo abandonaron por estrategias privadas de transporte. El estudio de las prácticas sociales de movilidad urbana cotidiana ha sido fundamental para comprender este fenómeno $\underline{\underline{2}}$, aplicándose un enfoque etnográfico con el fin de aprender sobre éstas.

Una de las principales técnicas de investigación aplicadas ha sido el sombreo, que constituye una forma de acompañar a los participantes de la investigación de manera individual en sus rutinas diarias, proceso durante el cual el/la participante sabe que está siendo acompañado/a mientras que el investigador observa la manera en quelleva a cabo sus prácticas cotidianas(Jirón, 2010). La interacción investigador-participante no está excluida durante dicho lapso de tiempo, pudiendo discutir ciertos temas durante el período de sombreo o permanecer en silencio. El viaje puede ser fotografiadoo filmado, dependiendo de las posibilidades de cada situación.

En base a este trabajo etnográfico, se puedenobservar tres principales maneras en que los viajeros han incorporadosus experiencias de viaje a sus prácticas cotidianas de movilidad: reaprender a viajar, aprender a evadir y aprender a usar el tiempo de espera. Estas tres prácticas han permeado el sistema de Transantiago, evidenciando las formas en que los usuarios se adaptan a éste pero también transformandosu aparente rigidez.

La escasa e insuficiente información disponible en torno a la implementación y funcionamiento de Transantiago obligó a los viajeros a reaprender a viajar por sí mismos en términos de cómo navegar a través del nuevo sistema, sin previo adiestramientoy con mínimas instrucciones (Jirón, Imilan \&Iturra, 2016). Al interactuar con su nuevo entorno en movimiento, los viajeros lo resignificaron y comenzaron un proceso de lugarización, aumentando su apropiación y significación a medida que iban adueñándose del sistema, apropiándose de él por medio de su conocimiento encorporado.

El proceso de reaprender a viajar ha implicado en buena medida una forma de disciplinamiento del viaje. Por ejemplo, hacer fila para subirse al bus, una práctica que previo a Transantiago no existía, ya que las personas se aglomeraban en la puerta del autobús esperando poder subirse de alguna forma. Con Transantiago, hubo la necesidad de armar filas distintas para aquellos que deseaban sentarse y otras para aquellos dispuestos a viajar parados. Aunque ésta es una práctica de la que no se habla, las personas saben que deben esperar en la fila cuando ya se han completado los asientos para así tener prioridad de ir sentados cuando parta el siguiente bus. Esta misma práctica puede observarse también en las estaciones de metro, donde las personas prefieren esperar en fila la llegada del siguiente carro.

Otro ejemplo se vincula al sistema de metro, el cual aumentó considerablemente su uso desde su incorporación al Transantiago. Debido a esto, las autoridades han tenido que diseñar estrategias para minimizar las aglomeraciones en los andenes de acceso a los vagones y en elinterior de éstos. 
Algunas líneas han implementado "estaciones rojas y verdes”, donde los trenes sólo paran en los colores designados y los pasajeros sólo se suben en los trenes del color de la estación de destino. Esto ha significado una espera y un abordaje más ordenado a los trenes, permitiendo a los pasajeros esperar en fila por la llegada de su vagón respectivo. Esto no significa que actualmente no haya aglomeraciones significativas en el metro, donde en ocasiones surge la necesidad de cerrar estaciones para no generar excesiva aglomeración de viajeros en su interior. Sin embargo, quienes quedan fuera de ellas saben que esto es sólo una situación momentánea y que las puertas volverán a abrirse lentamente.

Otra de las prácticas cotidianas que aún se mantiene pese a los cambios del Transantiago es el pago del boleto por parte de algunos de los viajeros que se suben por las puertas traseras del autobús. Previo al Transantiago, cuando los pasajeros se subían por las puertas traseras del autobús, muchos -no todos-, hacíanpasar su dinero de mano en mano entre los otros pasajeros quienes se la hacían llegar al chofer para pagar por el boleto. Los demás viajeros sabían que cuando un viajero les entregaba dinero pidiendo pagar, era necesario "hacerlo correr". Al poco tiempo, los mismos pasajeros circulaban el boleto de regreso, muchas veces acompañado por el vuelto para el pasajero pagante. Esta práctica daba cuenta de un nivel de confianza entre viajeros sabiendo que el pasaje seríapagado y que el pago no se perdería en el camino. Hoy en día esta práctica ya no existe, ya que no existe pago de pasaje con dinero. Lo que sí se evidencia hoy es la recreación de esa antigua práctica de circulación del pago con la tarjeta Bip!, ya que algunos pasajeros que se suben por las puertas de atrás hacen correr la tarjeta a través de los otros viajeros, quienes la acercan al validador automático y luego devuelven la tarjeta a su dueño.

Aprender a viajar no solo ha sido una experiencia de disciplinamiento para los viajeros, ya que muchos generalmente desafían el sistema como es el caso con la evasión de tarifa. Las tasas actuales de evasión en los buses del Transantiago es superior al 30\% (Gallegos et al., 2015), una tasa desproporcionada comparadacon las de otras ciudades (Tirachini et al., 2016). De acuerdo con estos autores, la evasión de tarifa en los buses de Transantiago se ha duplicado desde su origen, y actualmente amenaza la sustentabilidad financiera del sistema.

A lo largo de los años, muchos pasajeros de Transantiago aprendieron que era más beneficioso no pagar por el pasaje considerando las mínimas desventajas y sanciones que ello comporta, y en ese entendido han diseñado formas de evasión total o parcial del pago. Las razones parano pagar son muchas: algunos mencionan necesidad económica, ya que la tarifa ha aumentado considerablemente desde el inicio del nuevo sistema, elevando en exceso el costo si tienen que tomar los buses varias veces al día.

En el contexto de una ciudad extremadamente desigual, la evasión tarifaria también puede estar asociada a falta de recursos para pagar por el transporte público. Según Tirachini (2016), los viajeros pertenecientes al primer quintil, gastan por sobre el 20\% de sus ingresos en transporte, una proporción demasiadoalta para muchos. Muchos pueden pagar el viaje solo de ida, pero buscan formas de no pago para el viaje de vuelta, o viceversa. Otros mencionan la mala calidad del servicio como su principal razón para no pagar, quejándose por la baja frecuencia, el deteriorado estado de los buses, el hacinamiento al interior de éstos, y la lentitud de los recorridos, entre otras causas. En tal sentido, la situación de incertidumbre que comporta cada viaje es un tema central para sentirse 
insatisfecho con el sistema, incertidumbre que se expresa en situaciones como no saber la duración del viaje debido a la congestión de las vías, o también la incertidumbre frente a la hora de llegada de los buses a cada paradero.

Las estrategias de evasión son muchas, e incluyen entrar por las puertas traseras, argumentar falta de crédito en la tarjeta BIP!, pedir permiso al chofer para pasar, aprovechar la entrada de aglomeraciones o simplemente hacerse el desentendido. Al utilizar la tarjeta BIP!, los pasajeros cuentan con dos horas de tiempo protegido para conectarse con otro bus o metro una vez en el sistema, y muchosesperan llegar al metro para efectivamente pagar, y si no usan el metro del todo, simplemente no pagan. Los choferes de buses ignoran a los viajeros que no pagan, evitando así cualquier conflicto con los pasajeros, ya que su sueldo no depende del pago de la tarifa por parte de éstos. Algunos choferes mencionan que son las propias empresas de buses que les dicenque no intenten cobrar, que no vale la pena. El único temor de los pasajeros evasores es el inspector fiscalizador. Muchos reconocen a los fiscalizadores y alos lugares donde están ubicados, y buscan formas de evitarlos. Asimismo, aplicaciones móviles creadas para informar la ubicación de los fiscalizadores facilitanla práctica de evasión por parte de los pasajeros.

Pese a que la evasión es un tema muy relevante para el servicio Transantiago, el Ministerio de Transporte y Telecomunicaciones (MTT) aun no realiza un estudio serio sobre las razones y las prácticas de evasión. Sinembargo, se han implementado múltiples estrategias para obligar a los viajeros a pagar, incluyendo complejos torniquetes, más inspectores, aumento de tarifa, aumento de multas, entre otras.

No obstante estas medidas, resulta fundamental observar y estudiar las estrategias de evasión utilizadas por los pasajeros para comprenderlas en profundidad. Al ser la evasión una práctica cada vez más generalizada entrelos viajeros, pareciera que ella expresa una problemática mayor que no es considerada por las autoridades. Algunos viajeros, particularmente los más jóvenes, mencionan que la evasión posee incluso un carácter político al constituirse en una forma de protesta frente a los múltiples problemas presentes en el país, como son la distribución inequitativa de la riqueza, casos de corrupción, colusión, entre otros. En tal sentido, y entendiendo que la estrategia de perseguir a viajeros evasores podría ser una tarea muy difícil y costosa, la comprensión de la práctica de evasión podría aportar al diseño de soluciones alternativas más efectivas.

Otro de los problemas asociados a Transantiago ha sido la necesidad de aprender a usar el tiempo de viaje. Previo a la implementación del sistema, era posible cruzar buena parte de la ciudad de Santiago en un mismo bus, con el consecuente pago de un solo boleto y sin necesidad de realizar intercambios. Hoy, el sistema troncal-alimentador involucra un número importante de estaciones de trasbordo por toda la ciudad. Muchos se quejan de tener que hacer transferencia entre buses o de buses a metro, con el consecuente costo en términos de tiempo y dinero.

En este contexto, ha surgido una oportunidad para vendedores informales quienes utilizan estas estaciones de intercambio y aprovechan el tiempo de espera de los pasajeros para vender desayunos, almuerzos, onces o snacks que van desde sándwiches, fruta, jugos, te, café, sopaipillas ${ }^{\underline{3}}$ en el invierno o refrescante mote con huesillos ${ }^{4}$ en verano. El tiempo que las personas pasan en las zonas de transferencia son utilizadas para comer, mezclarse con otros pasajeros e iniciar conversaciones 
para informarse de las últimas novedades, entre otras prácticas. Estás prácticas aparecen asociadas a un aumento del consumo de comida chatarra entre viajes.

En medio del trajín matutino, el aumento de los tiempos de viaje -ya sea por los tiempos extras de espera o por la dificultad de subirse al autobús debido a las aglomeraciones-significa muchas veces que los viajeros necesitan levantarse más temprano y saltarse el desayuno en casa. Es común ver a personas comiendo en los buses y, más común aún, verlos parar en su camino al trabajo para comprar desayuno de los vendedores ambulantes. En las tardes, muchos formas filas para comprar snacks fritos camino a casa. Y a lo largo del día, los puestos de comida son una manera fácil de pasar el rato, apagar la sed y calmar el hambre. Pese a que los puestos de comida informal son comunes en ciudades latinoamericanas, en Santiago ha habido un aumento de los diversos tipos de vendedores ambulantes en los últimos años, particularmente en áreas de trasbordo de Transantiago. En un contexto de altas tasas de sobrepeso y obesidad entre la población chilena (Encuesta Nacional de Consumo Alimentario - ENCA, 2014), particularmente engrupos de menores ingresos, queterminan comiendo calorías baratas, el aumento de comida informal, particularmente en las zonas de trasbordo de Transantiago, es importante ya queun tercio de la obesidad prevalente se debe a causas individuales y dos tercios a entornos alimenticios (Mooney et al., 2011). Este es un tema de particular interés futuro en Chile.

\section{Reflexiones Finales}

A la luz de los resultados de investigación anteriormente descritos, es posible sintetizar la relevancia de las prácticas sociales en el estudio de la movilidad urbana cotidiana en tres grandes consideraciones.

La primera consideración indica que las prácticas sociales constituyen un componente fundamental de los aprendizajes y competencias que los habitantes urbanos adquieren para adaptarse a las transformaciones y habitar sus ciudades. Los tres ejemplos de formas en que los viajeros urbanos aprenden y aprovechan el Transantiago dan cuenta de cómo las prácticas de movilidad son una parte muy importante de la vida cotidiana de los viajeros, y de su relevancia para la implementación de una política de transporte en la ciudad. El disciplinamiento en el acceso y uso de los buses, la evasión de pago, o la alimentación en los fragmentos espacio-temporales que otorga el sistema son interesantes ejemplos de la manera en quelos usuarios del Transantiago se han adaptado y al mismo tiempoadaptado el sistema a lo largo de los años.

La segunda consideración indica que las prácticas sociales son relevantes para producir nuevos conocimientos y saberes integrados entre habitantes y planificadores urbanos. La estrecha relación entre prácticas sociales y cotidianeidad permite visibilizar y problematizar diversos aspectos de la vida cotidiana de los habitantes urbanos que muchas veces son desconocidos, invisibilizados e ignorados por aquellos actores sociales que diseñan e implementan políticas y programas urbanos como Transantiago.

La consideración de un enfoque de prácticas sociales para abordar los procesos urbanos conlleva la invitación a desarrollar una perspectiva de conocimiento que vaya más allá de los expertos, que 
incorpore e involucre otros actores y agentes sociales, depositarios de otros saberes y otras formas de producir conocimiento.En este sentido, la producción de conocimiento sobre ciudad y territorio debe desarrollarse como un proceso dinámico que conciba un problema integral, reconociendo sus múltiples dimensiones - política, administrativa, social, etc.- y que desarrolle propuestas integradas en distintas escalas - vivienda, barrio, ciudad, territorio.

La tercera consideración indica que las prácticas sociales son relevantes para cocrear. La diversidad de conocimientos y saberes que conlleva la visibilización y reconocimiento de las prácticas sociales urbanas permite pasar desde un enfoque de interacción entre expertos y habitantes a uno de cocreación.

La cocreaciónse entiende como un proceso donde nuevas ideas son diseñadas en conjunto con las personas, y no para ellas (Waissbluth et al., 2014), surgiendo fundamentalmente de la necesidad de cambios, ya sea a través de las tecnologías de entrega de servicios, comunicaciones o los patrones de entrega de servicios(Jirón, 2016). En el ámbito urbano, versiones iniciales de la cocreación se pueden detectar a partir de la planificación colaborativa en Inglaterra (Healey, 1997 y 2003) como una forma alternativa de la planificación urbana tradicional de arriba hacia abajo, con el fin de empoderar a los ciudadanos a jugar un rol más significativo en la planificación de su espacio.

En tal sentido, su relevancia radica justamente en el principio de que el conocimiento no proviene sólo de expertos, como arquitectos, diseñadores urbanos o urbanistas sino que también se nutre del conocimiento local que los habitantes urbanos producen a través de sus prácticas sociales cotidianas. Pasar de la planificación urbana o de transporte tradicional a la cocreación requiere que los planificadores pierdan un poco el control en su quehacer y distribuyan el poder en la toma de decisiones del proceso, permitiendo la intervención, la participación y el involucramiento de los habitantes, independientemente de su contexto social o profesional.

\section{Notas}

1 Para una revisión detallada sobre los problemas de implementación de Transantiago, ver Jirón (2012).

$\underline{2}$ El presente trabajo se basa en cuatro investigaciones sobre prácticas de movilidad en la ciudad de Santiago de Chile. El primero fue realizado entre los años 2005 y 2007, previo a la implementación de Transantiago, y se refiere a las prácticas de movilidad de 12 familias que habitan el sector suroriente de Santiago (Jirón 2009 y 2010). El segundo se realizó entre los años 2009 y 2012, post Transantiago, y en él se sombrearon las prácticas de movilidad de 76 casos en diversos sectores de la ciudad de Santiago (Jirón e Imilan, 2016). El tercero fue parte de una investigación más amplia sobre estrategias de movilidad, y se realizó entre los años 2014 y 2017 e incluyó el sombreo de 10 viajeros en el sector norte de la ciudad de Santiago. Finalmente, como parte del curso de Etnografía urbana de la Carrera de Geografía de la Universidad de Chile, se etnografiaron las prácticas de evasión en 12 líneas de buses de Transantiago.

3 Típica comida chilena consistente en una masa de zapallo frita.

4 Típico brebaje frío chileno consistente en un jugo de durazno con mote. 


\section{Bibliografía}

Alamo, C. (2008). Pérez Yoma Edmundo: 'Hay que estar preparado para perder el poder'. Revista Cosas, 32, 42-45, 17 julio 2008.

Barthe, Y., Rémy, C., Trom, D., Linhardt, D., De Blic, D., Heurtin, J.... (2016). Sociología Pragmática: guía do usuario. Sociologías, 18(41), 84-129.

Bourdieu, P. (1996).Cosas Dichas. Barcelona: Ed. Gedisa

Corcuff, P. (2016). Las Nuevas Sociologías. Principales corrientes y debates, 1990-2010. Buenos Aires: Siglo XXI.

Cresswell, T. (2010). Towards a politics of mobility. Environment and Planning D: Society and Space, 28(1), 17-31.

de Certeau, M. (1986).The Practice of Everyday Life. London: University of California Press.

ENCA (2014). Encuesta Nacional de Consumo Alimentario. Informe final. Santiago de Chile: Universidad de Chile. Recuperado dehttp://web.minsal.cl/sites/default/files/ENCAINFORME_FINAL.pdf.

Gallegos, N., Munizaga, M.\&Gschwender, A. (2015). Factores de expansión por evasión para matriz OD de viajes en transporte público. Actas XVII Congreso Chileno de Ingenieria de Transporte, SOCHITRAN 2015. Concepción, 12-16 octubre 2015. Recuperado de http://www.sochitran.cl/wp-content/uploads/Acta-2015-01-10.pdf.

Healey, P. (1997). Collaborative Planning: Shaping places in fragmented societies. London: Macmillan.

Healey, P. (2003). Collaborative Planning in Perspective.Planning Theory,2(2), 101-123.

Jirón, P. (2009).Mobility on the move: Examining urban daily mobility practices in Santiago de Chile(PhD Thesis), London School of Economics and Political Science, London, UK.

Jirón, P. (2010). On becoming 'la sombra/the shadow'.En Büscher, M., Urry, J. \& Witchger, K. (eds),Mobile Methods(pp. 36-53).New York: Routledge-.

Jirón, P.\&Imilan, W. (2016). Posibilidades, desafíos o encrucijadas de una 'etnografía colectiva': Propuesta metodológica para el estudio de la movilidad cotidiana urbana. Revista Alteridades,26(52), 51-64.

Jirón, P., Imilan. W.\&Iturra, L. (2016). Relearning to travel in Santiago: the importance of mobile place-making and travelling know-how. Cultural Geographies,23(4), 599-614.

Jirón, P. \& Imilan W. (2016). Observando juntos en movimiento: posibilidades, desafíos o encrucijadas de una 'etnografía colectiva'.Revista Alteridades,52.

Jirón, P. (2016). De la participación a la co-creación. Nuevas formas de pensar intervenciones para mejorar el habitar urbano/residencial.Revista Materia Arquitectura, 12. 
Jirón, P. \& P. Mansilla (2014). Las consecuencias del urbanismo fragmentador en la vida cotidiana de habitantes de la ciudad de Santiago. Revista EURE - Revista De Estudios Urbano Regionales, 40(121).

Lefebvre, H. (1972).La Vida Cotidiana en el Mundo Moderno. Madrid: Alianza.

Nardacchione, G. (2016). Laurent Thévenot, autor de una sociología heterodoxa en la Francia del siglo XX. En Thévenot, L.,La acción en plural. Una introducción a la sociología prágmática. 1 edición(pp. 11-24). Buenos Aires: Siglo XXI.

Mooney, J., Haw, S.\& Frank, J. (2011). Policy Interventions to Tackle the Obesogenic Environment: Focusing on adults of working age in Scotland. Medical Research Council and the Chief Scientist Office of the Scottish Government, Scotland.

MOPTT (2004). Plan de transporte urbano de Santiago, Chile: Transantiago súbete. Santiago, Chile: Ministerio de Obras Públicas, Transporte y Telecomunicaciones, Gobierno de Chile.

Reckwitz, A. (2002). Towards a theory of social practices: a development in culturalist theorizing'. European Journal of Social Theory,5, 243-263.

Schatzki, T. (1996).Social Practices: A Wittgensteinian Approach to Human Activity and the Social. Cambridge:Cambridge University Press.

Schatzki, T. (2001). Introduction: practice theory. En T. R. Schatzki, K. Knorr Cetina \& E. von Savigny (Eds), The practice turn in contemporary theory(pp. 1-14). London: Routledge.

Schatzki, T. (2002). The site of the social: a philosophical account of the constitution of social life and change. University Park, PA: Pennsylvania State University Press.

Schatzki, T. (2012). A primer on practices. En J. Higgs, R. Barnett, S. Billett, M. Hutchings \& F. Trede (Eds),Practice-based education: perspectives and strategies(pp. 13-26). Rotterdam: Sense.

Thevenot, L. (2016). La acción en plural. Una introducción a la sociología prágmática. 1 edición. Buenos Aires: Siglo XXI.

Tirachini, A.\&Quiroz, M. (2016).Evasión del pago en transporte público: evidencia internacional y lecciones para Santiago. Documento de Trabajo. Santiago: Departamento de Ingenieria Civil, Universidad de Chile.

Ureta, S. (2015).Assembling Policy. Transantiago, Human Devices and the Dream of a World-Class Society. Cambridge:The MIT Press.

Vaiou, D. \&Lykogianni, R. (2006). Women, neighbourhoods and everyday life. Urban Studies, 43(4), 731-743.

Warde, A. (2004). Practice and field: revising Bourdieusian concepts. CRIC Discussion Paper No 65. Centre for Research on Innovation \& Competition, 1-35. Manchester: The University of Manchester.

Warde, A. (2005). "Consumption and Theories of Practice." Journal of Consumer Culture,5(2), 131153. 
Weissbluth , M., E. Contreras, P. Galaz, I. Aguilera, J. Inostroza, A. Infante, ... M. Gatica (2014). Co-creación para la Innovación: Un caso en el Sector Público Chileno, Revista Ingeniería de Sistemas, XXVIII. 